\title{
Ten years of anti-HPV vaccinations: what do we know?
}

\author{
Robert Jach ${ }^{1}$, Antoni Basta ${ }^{2}$, Jan Kotarski ${ }^{3}$, Janina Markowska ${ }^{4}$, Tomasz Paszkowski ${ }^{5}$, Romuald Dębski \\ Wojciech Rokita ${ }^{7}$, Witold Kędzia ${ }^{8}$, Krystyna Kiszka ${ }^{9}$
}

${ }^{1}$ Clinic of Endocrynologic Gynecology, University Hospital UJ CM, Krakow, Poland

${ }^{2}$ Clinic of Gynecologic Oncology, University Hospital UJ CM, Krakow, Poland

${ }^{3}$ First Clinic of Gynecologic Oncology and Gynecology, Medical University of Lublin, Lublin, Poland

${ }^{4}$ Clinic of Oncology, Poznan University of Medical Sciences, Poznan, Poland

${ }^{5}$ III Chair and Clinic of Gynecology, Medical University of Lublin, Lublin, Poland

${ }^{6}$ Department of Gynecology and Obstetrics CMKP, Bielański Hospital, Warsaw, Poland

${ }^{7}$ Clinic of Gynecology and Obstetrics, WSZ, Kielce, Poland

${ }^{8}$ Clinic of Gynecology, Gynecologic-Obstetrical Clinical Hospital UM in Poznan, Poland

${ }^{9}$ Department of Gynecology and Obstetrics SP ZOZ in Myślenice, Poland

\begin{abstract}
Human papillomavirus (HPV) is one of the most important carcinogens in humans. Vaccines against HPV are now considered the first anti-cancer vaccinations. Since 2007, in many developed countries, there have been recommendations present for preventive vaccines against HPV. At present, the degree of implementation of these recommendations depends on a number of country-specific factors such as the health care system organization or the ways of funding. HPV vaccines are primarily to prevent the development of cervical cancer and other genital cancers. Therefore, only their long-term effectiveness can be measured, when a correspondingly large cohort of vaccinated teenagers reaches the age of the greatest incidence of these cancers. However, great care should be taken in assessing the results of vaccinations due to the possibility of misinterpretation and possible erroneous data.

Undoubtedly, teenagers are the target population of HPV vaccines. However, vaccinating young sexually active women is also justified from an individual point of view. A 9-valent vaccine has been registered in the USA and in Europe - including Poland - as one of the three preventive vaccines. It is recommended to vaccinate women between 13 and 26 and men between 13 and 21, previously unvaccinated. It is also recommended to vaccinate men aged 26 years or less who have sexual relations with other men and people with reduced immunity, including HIV-positive people who have not been vaccinated previously.
\end{abstract}

Key words: HPV, human papillomavirus, HPV vaccine.

\section{Introduction}

Human papillomavirus (HPV) is one of the most important carcinogens in humans, classified in Group 1, carcinogenic to humans, of the International Agency for Research on Cancer (IARC).

The introduction of two prophylactic vaccines that can prevent HPV infections and the development of precancerous lesions and invasive cancers have opened

Tab. I. The proven role of HPV infection in malignant neoplasms among women [1]

\begin{tabular}{lc}
\hline All cancers in women (in developing countries) & $16 \%$ \\
\hline All cancers in women & $10 \%$ \\
\hline All cancers & $5 \%$ \\
\hline
\end{tabular}

new perspectives in vaccinology. Vaccines against HPV are now considered the first anti-cancer vaccines (though, in fact, the first vaccines of this profile are vaccines against hepatitis $B$ ).

Due to the inability to measure the direct effect of anti-cancer vaccines for ethical reasons and time constraints of clinical observations, it was assumed that the end-point protection surrogate will be preventing cervical intraepithelial neoplasia grade 2/3 (CIN2+). The clinical tests of both (2-valent and 4-valent) vaccines assumed the prevention of $\mathrm{CIN} 2+[2,3]$ as the primary end-point.

Since 2007, in many developed countries, there have been recommendations for preventive vaccinations against HPV. At present, the degree of implementation of these recommendations depends on a number of country-specific factors, such as the health care system organization or the ways of funding. 


\section{Effectiveness of vaccinations against HPV}

Human papillomavirus vaccines are primarily to prevent the development of cervical cancer and other genital cancers. Therefore, only their long-term effectiveness can be measured when a correspondingly large cohort of vaccinated teenagers reaches the age of the greatest incidence of these cancers.

Due to the fact that the transition of chronic HPV infections to precancerous lesions (CIN1-3) and to cancer lesions is well known, many models were designed for the influence of HPV vaccination programs on the incidence of diseases. This made it possible to predict both clinical and economic effects of vaccinations, prior to the availability of both vaccines on the market.

All of these models assumed, however, that universal vaccinations of girls combined with cytological screenings significantly affect the incidence of cervical cancer and will be favorable from an economic point of view [4]. Constantly, new publications are being developed on the impact of HPV vaccination strategies on the incidence of cervical cancer, which examine new target groups, including young boys. Another important element triggered by the past experience of anti-HPV vaccination is the alternative vaccination schedules reduced to two doses [5].

The result of the observations conducted is the knowledge on the impact of HPV vaccination on conditions with shorter natural history than in the case of cervical cancer, in particular, the comparison between vaccinated and unvaccinated patients with regard to the presence of chronic HPV infections, genital warts (the 4-valent vaccine) and precancerous lesions (CIN2+).

However, great care should be taken in assessing the results of vaccinations due to the possibility of misinterpretation and possible erroneous data. Monitoring the prevalence of HPV infections requires answering the question of collecting material, detecting and genotyping HPV from a variety of infected body sites and considering different screening strategies at the age of starting screening, screening interval and the diagnostic tool (HPV test). All of these elements can significantly affect the percentage of detectable HPV infections and dependent lesions [6]. Despite these reservations, however, more and more data from many countries provide evidence of the effectiveness of prophylactic vaccinations against HPV.

Undoubtedly, Australia is a country in which a vaccination program was introduced in the most comprehensive manner and where a high percentage of vaccinated young women was achieved in a short time. The proportion of those vaccinated with three doses is there, $30 \%$ and $70 \%$, respectively, for women in the age range of 20-26 and 12-13. In contrast, vaccination with at least one dose of the vaccine in the cohorts of women is at the level of $52 \%$ and $83 \%$ [7].

A comparative study of the incidence of HPV in the cytosmears of Australian women prior to the in- troduction of vaccination (2005-2007), and after the introduction of vaccination (2010-2011) showed that the prevalence of HPV vaccine types $(6,11,16,18)$ was significantly lower in the vaccinated cohort $(6.7 \%$ vs. $28.7 \%, p<0.001$ ). A lower prevalence of HPV infection was also recorded in both the vaccinated and unvaccinated female population, as compared to the population prior to the introduction of vaccination $(5.0 \%$ [OR: 0.11, Cl: 0.006-0.21] and 15.8\% [OR: 0.42, Cl: 0.190.93], respectively) [8].

The impact of vaccination on the incidence of genital warts is also obvious. In the Australian study evaluating trends in the occurrence of genital warts in 20042011, involving more than 85000 participants, a huge decrease was observed in the proportion of women under 21 and at the age of 21 to 30 who were diagnosed with genital warts, from $11.5 \%$ in 2007 to $0.85 \%$ in 2011 , respectively (a decrease by $92.6 \%, p<0.001$ ) and $11.3 \%$ in 2007 to $3.1 \%$ in 2011 (a decrease by $72.6 \%$, $p<0.001)$. There was however no decrease recorded in the incidence of genital warts in women over 30 years of age. An indirect effect of the immunization of young women population was their reduced incidence of genital warts, $81.8 \%$ and $51.1 \%$, respectively, in the population of young heterosexual men (under 21 and at the age of 21-30). There was no decrease in the incidence of genital warts in men over 30 [9].

A third Australian study by Brotherton et al. showed a decrease in high-grade epithelial lesions in the cervix (CIN2 + and AIS - adenocarcinoma in situ) among vaccinated women below 18 covered by the vaccination program, as compared with the period before the introduction of population-based vaccination (2003-2007) to the period of 2007-2009 (post-vaccination). There has been a $50 \%$ decrease in the incidence of CIN2+ and AIS ( $p=0.003$ ) from $0.85 \%$ in 2006 to $0.22 \%$ in 2009 [10].

Evidence of the protective effect of vaccination against HPV also comes from the Nordic countries. A study carried out in Denmark demonstrated that while the incidence of genital warts was $2 \%$ in the period before immunization, the condition was absent after the introduction of vaccination in the population (population coverage was $85 \%$ ) of females aged $16-17$. There was no indirect effect of the vaccination of girls on the incidence of genital warts in young men [11].

In all of the Nordic countries, a long-term follow-up program was introduced for women covered by the program of preventive vaccination against HPV. Because all of the women in the placebo group in the Future II study were vaccinated after 60 months for ethical reasons, it is not currently possible to determine the effects of vaccination [12]. It should be noted, however, that within 8 years of the observation there was no case of CIN2+ associated with HPV 16/18 in women from the group vaccinated according to the protocol of vaccination [13]. 
In the USA, the degree of vaccinating girls aged (13-17) remains low (32\% in 2010). Nevertheless, data from the National Health and Nutrition Examinations Surveys clearly show (56\%) a decrease in HPV infections with compact types in the case of vaccinated girls aged 14-19 years, comparing the periods 2003-2006 to 2007-2010). The efficacy of vaccination (assuming at least one dose) was rated at $82 \%$ [14].

Studies of the vaccine against HPV have shown varying degrees of cross-immunity against the types of the virus not present in the vaccines. Such an unexpected mechanism was observed, e.g. in the PATRICIA study (Papilloma Trial Against Cancer in Young Adults) evaluating the efficacy of a bivalent vaccine. The efficacy of the vaccine was demonstrated with respect to the types of HPV that cause the formation of genital warts. The effectiveness of the 6-month vaccine was 34.5\% against HPV 6/11, 34.9\% against HPV 6 and 49.5\% against HPV 74 (all statistically significant). Unfortunately, in the long-term perspective, it was observed that the cross-immunity begins to fade with time, and after a period of 6.4 years and 8 years, there was no statistical significance [15]. In the UK, the vaccination program consisted of girls aged 12 (reaching $80 \%$ coverage of the population and an additional $40 \%$ in the revaccination program to 18 years old). Reducing the incidence of genital warts was $11 \%$ to $16 \%$ in women aged 18 and 15 .

Human papillomavirus is associated with a risk of not only lesions in the genital area, but also a significant proportion of cancers of the head and neck. The Centre for Disease Control and Prevention (CDC) envisages a significant effect of prophylactic vaccination on the reduction of morbidity and mortality from cancers of the head and neck [16].

It should be remembered that in a small percentage of the cases of cancer of the vulva, vagina, anus, and especially penis, DNA HPV 6 is recorded, from the lowrisk group [17].

Observational studies are also ongoing with respect to the possibility of replacing the niche left by the HPV types included in the vaccine by other HPV types. However, due to the characteristics of HPV (among other things, the possibility and incidence of multiple infections), it is now unlikely that such a phenomenon occurred.

The possibility of reducing the doses of the vaccine entails several advantages. First of all, it enables an increase in the population covered by the vaccination and results in its cost reduction, which can be used, e.g. to extend the cohorts undergoing immunization.

Two studies of the effectiveness of the two-dose immunization schedule (Romanowski et al. 2011 - I/II phase study (HPV048) and Puthanakit et al. 2013 - III study phase (HPV-070) demonstrated clinical efficacy $[18,19]$. With regard to the conditions associated with HPV 16 and 18 , the two-dose immunization vaccination schedule for girls aged 9-14 was not immunologically less effective than the three-dose schedule in the population of girls aged 15-25 [20]. The immune response 48 months after vaccination with two doses was still at a high level [21]. The European Medicine Agency (EMA) approved, in 2013, the two-dose bivalent vaccination of girls aged 9-14.

For the 4-valent vaccine, there were no ad hoc studies planned to assess the change of the vaccination schedule of young girls. Nevertheless, in some countries, independently, studies were conducted on the off-label use of the vaccine in the two-dose schedule. The study conducted in Canada on a population of 830 girls stratified according to their age showed no inferiority of the two-dose schedule over the three-dose one [22]. In 2014, the EMA issued a positive opinion on the possibility of adapting the 4-valent vaccine to the twodose schedule $(\mathrm{MO}, 6)$ in girls aged 9-13.

In June 2015, the first reports were published on the effectiveness of preventive vaccination with the one-dose schedule, particularly in developing countries [23]. So far, 45 countries in the world (mainly developed) have introduced a population prophylactic vaccination program.

Despite the efficacy and safety profile of prophylactic vaccines against HPV, the social acceptance of vaccination and the percentage of vaccinated populations remain far from optimal. According to data from the European Centre for Disease Prevention and Control of Diseases (ECDC), there are several reasons for such a state of affairs. The most important ones seem to be: low knowledge of HPV vaccines and prophylactic vaccines, high cost of vaccination, low expected efficiency of vaccines, and alleged and actual side effects of vaccines. An important barrier is the fear of parents whether vaccinations increase the promiscuity and risky sexual behaviors of their children. From this point of view, the paper by Bednarczyk et al. is important; it did not show an increased percentage of sexual activity in girls immunized at the age of 11-12, observed for three years [24].

Undoubtedly, teenagers are the target population of HPV vaccines. However, vaccinating young, sexually active women is also justified from an individual point of view [25]. First of all, the risk of infections with all four HPV types present in the vaccines is negligible $(<1 \%)$, secondly, the vaccines are effective against the HPV types with which the woman was not infected at the time of immunization and, finally, there is evidence that vaccines provide protection against the HPV types that were cured (seropositive but DNA-negative). All sexually active women are constantly exposed to HPV infections.

As far as vaccinating men is concerned, it was shown that prophylactic vaccinations against HPV are highly (90\%) effective in the prevention of condyloma acuminata [26]. Efficacy against anal intraepithelial neoplasia is estimated to be $92 \%$ and, therefore, the vaccine would be the most effective in homosexual men [27]. 


\section{Organizational experience with respect to anti-HPV vaccinations}

The guidelines for the use of vaccines developed by the European Centers for Disease Prevention (ECDC) include detailed information on the legitimacy of their use, efficiency, safety, and the suggested target group. They also recommended monitoring vaccination programs, which have been implemented in some countries. Previous studies on HPV vaccination programs in Europe helped to create a map of areas covered by the vaccination programs and their structure. The VENICE study showed large differences between the programs and focused on the decision-making processes for the implementation of the programs. Studies conducted in Central and Eastern Europe also provided information on the programs there, the incidence of HPV and the distribution of different types of the virus, which is a preliminary requirement to assess the impact of vaccinations. The studies provided detailed information on how the programs developed, how they were implemented, and they emphasize the role of knowing the structure of infections in a given country prior to starting the program.

Vaccination programs against HPV require financial and personal involvement. To achieve the expected results, there is a need for continued monitoring, evaluation and optimization of the activities conducted. Combining medical records enables continuous surveillance of the key data collected during screening, the incidence of cancer registers and the records of immunization: immunization coverage, the rate of unfinished vaccination cycles, the incidence of CIN, cancer, genital warts and HPV infections, as well as side effects of vaccines. Such connection of databases, however, requires a substantial contribution to the assessment of individual epidemiological phenomena and the structure of the overall protection of personal data. Furthermore, registers should be interconnected at the level of individual records, which would enable demonstrating direct relationships of a particular case in individual databases. As the participants of the first study conducted get older, it will be possible to assess the long-term impact of vaccinations.

\section{Prospects for HPV vaccination}

The nine-valent vaccine was registered by the FDA on December 10, 2014 for use in women of 9 to 26 and men from 9 to 15 [28].

The Advisory Committee on Immunization Practices (ACIP), in February 2015, recommended the use of the 9-valent vaccine as one of three possible vaccines against HPV. Recommendations for vaccinations against HPV indicate the population of 11- or 12-year-olds. ACIP recommends vaccinations of women between 13 and
26 and men between 13 and 21 , previously unvaccinated. It is also recommended to vaccinate men aged 26 or less who have sexual relations with other men and people with reduced immunity, including HIV-positive people who have not been vaccinated previously.

The 9-valent vaccine, just as the 4-valent one, contains a VLP (a virus-like antigen) for HPV 6, 11, 16, 18. Additionally, it contains a VLP for HPV 31, 33, 45, 52 and 58 [29].

In 2013-2015, an ACIP working group analyzed the clinical efficacy, immunogenicity and safety of the 9-valent vaccine. The recommendations were considered as category A [30].

\section{Efficacy, safety and immunogenicity of the 9-valent vaccine}

The III phase of the study compared the efficacy of the 4- and 9-valent vaccines in 14 thousand women aged 16-26. The efficacy of the 9-valent vaccine in preventing CIN2+, VIN 2 and 3, VAIN 2 and 3 caused by HPV $31,33,45,52$ and 59 was $96.7 \%$ (women vaccinated with three doses within a year, seronegative prior to the first dose, still seronegative one month after the third dose) [29, 31]. Efficacy against CIN2+, HPV 31, 33, 45, 52 and 58 was $96.3 \%$ and $96 \%$ for a persistent infection in a six-month observation period [31]. Most infections in both treatment groups were caused by HPV 6, 11, 16, 18. The immunogenicity of the 9-valent vaccine, comparable to the 4-valent vaccine, was used to demonstrate the efficacy of the vaccine in preventing HPV $6,11,16$, 18. The geometric mean titers of antibodies one month after the third dose were satisfactory for HPV 6, 11, 16, 18,2 in the group vaccinated with the 9-valent vaccine seroconversion rate of $99 \%$ was found for all 9 subtypes of HPV.

The 9-valent vaccine immunogenicity assessment was made in 2.4 thousand women and men from 9 to 15 and 400 women aged 16-26. The seroconversion rate of $99 \%$ was found for all 9 types of HPV, but the mean titers of antibodies were significantly higher in younger women.

A study comparing the immunogenicity of the 4-valent and the 9-valent vaccines in 600 women aged 9 to 15 showed the seroconversion rate for HPV 6, 11, 16, 18 of $100 \%$ in both groups, and mean titers of antibodies in the 9-valent group did not differ from those found in the 4-valent group.

Another study examined the immunogenicity of the 9-valent vaccine in men from 16 to 26 years of age, as compared with women in the same age range. The seroconversion rate of over $99 \%$ was found in both groups for all 9 types, the mean titers of antibodies were comparable [32].

Research on the 9-valent vaccine immunogenicity was assessed in a simultaneous combination with other vac- 
cines: the 4-valent conjugate vaccine against the meningitis (Menactra) and vaccines against tetanus, diphtheria and pertussis (Adacel). All groups reported titers of antibodies comparable to the mean. The safety of 9-valent vaccines was assessed in 4 studies under the auspices of the FDA [32]. The vaccine was well tolerated, and most of the side effects were associated with pain on injection, pruritus and rash. The severity of these ailments was mild or moderate. The safety profile was similar for the 4- and 9-valent vaccines.

\section{Contraindications and precautions}

Human papillomavirus vaccines are contraindicated in patients with previously demonstrated hypersensitivity to vaccine components. The 4 - and 9-valent vaccines are contraindicated in patients who are allergic to yeast. The 2-valent vaccine should not be used in people who are allergic to latex.

The vaccine is not recommended in pregnant women [33]. In women vaccinated with the starting dose, who subsequently became pregnant, subsequent doses should be postponed until the end of pregnancy. Pregnancy tests do not need to be performed in women prior to vaccination. If the vaccination took place during pregnancy, there is no need for intervention. New guidelines have been established for the 9-valent vaccine in pregnancy [29]. Registrations for pregnant women regarding the 4- and 2-valent vaccines were closed after the intervention of the FDA [33, 34]. Exposure to vaccination during pregnancy should be reported. Screening for cervical cancer is recommended for women over 21 and continued to the age of 65 for all vaccinated and unvaccinated women [35].

\section{Disclosure}

Authors report no conflict of interest.

\section{References}

1. de Martel C, Ferlay J, Franceschi S, et al. Global burden of cancers attributable to infections in 2008: a review and synthetic analysis. Lancet Oncol 2012; 13: 607-615.

2. Garland SM, Hernandez-Avila M, Wheeler CM, et al. Future II Study Group. Quadrivalent vaccine against human papillomavirus to prevent high-grade cervical lesions. N Engl J Med 2007; 356: 1928-1943.

3. Paavonen J, Naud P, Salmerón J, et al. Efficacy of human papillomavirus (HPV)-16/18 AS04-adjuvanted vaccine against cervical infection and precancer caused by oncogenic HPV types (PATRICIA): final analysis of a double-blind, randomised study in young women. Lancet 2009; 374: 301-314.

4. Dasbach EJ, Elbasha EH, Insinga RP. Mathematical models for predicting the epidemiologic and economic impact of vaccination against human papillomavirus infection and disease. Epidemiol Rev 2006; 28: 88-100.

5. Jit M, Choi YH, Laprise JF, et al. Two-dose strategies for human papillomavirus vaccination: how well do they need to protect? Vaccine 2014; 32: 3237-3242.
6. Hariri S, Markowitz LE, Dunne EF, Unger ER. Population impact of HPV vaccines: summary of early evidence. J Adolesc Health 2013; 53: 679-682.

7. Gertig DM, Brotherton JM, Saville M. Measuring human papillomavirus (HPV) vaccination coverage and the role of the National HPV Vaccination Program Register, Australia. Sex Health 2011; 8: 171-178.

8. Tabrizi SN, Brotherton JM, Kaldor JM, et al. Fall in human papillomavirus prevalence following a national vaccination program. J Infect Dis 2012; 206: 1645-1651.

9. Ali $\mathrm{H}$, Donovan B, Wand $\mathrm{H}$, et al. Genital warts in young Australians five years into national human papillomavirus vaccination programme: national surveillance data. BMJ 2013; 346: f2032.

10. Brotherton JM, Fridman M, May CL, et al. Early effect of the HPV vaccination programme on cervical abnormalities in Victoria, Australia: an ecological study. Lancet 2011; 377: 2085-2092.

11. Baandrup L, Blomberg M, Dehlendorff $C$, et al. Significant decrease in the incidence of genital warts in young Danish women after implementation of a national human papillomavirus vaccination program. Sex Transm Dis 2013; 40: 130-135.

12. Olsson SE, Villa LL, Costa RL, et al. Induction of immune memory following administration of a prophylactic quadrivalent human papillomavirus (HPV) types 6/11/16/18 L1 virus-like particle (VLP) vaccine. Vaccine 2007; 25: 4931-4939.

13. Kjær S, Dillner J, Marshall B, et al. Long term effectiveness of GARDASILTM in the Nordic countries. In: Abstracts of the $28^{\text {th }}$ Papillomavirus Conference, San Juan (Puerto Rico). 30 November - 6 December 2012; 270.

14. Markowitz LE, Hariri S, Lin C, et al. Reduction in human papillomavirus (HPV) prevalence among young women following HPV vaccine introduction in the United States, National Health and Nutrition Examination Surveys, 2003-2010. J Infect Dis 2013; 208: 385-393.

15. Lehtinen M, Paavonen J, Wheeler CM, et al. Overall efficacy of HPV16/18 AS04-adjuvanted vaccine against grade 3 or greater cervical intraepithelial neoplasia: 4-year end-of-study analysis of the randomised, double-blind PATRICIA trial. Lancet Oncol 2012; 1: 89-99.

16. Brotherton JM, Gertig DM. Primary prophylactic human papillomavirus vaccination programs: future perspective on global impact. Rev Anti Infect Ther 2011; 9: 627-639.

17. de Sanjose S, Quint WG, Alemany L, et al. Human papillomavirus genotype attribution in invasive cervical cancer: a retrospective cross-sectional worldwide study. Lancet Oncol 2010; 11: 1048-1056.

18. Romanowski B, Schwarz TF, Ferguson LM, et al. Immunogenicity and safety of the HPV-16/18 AS04-adjuvanted vaccine administered as a 2-dose schedule compared with the licensed 3-dose schedule: results from a randomized study. Hum Vaccin 2011; 7: 1374-1386.

19. Puthanakit T, Schwarz T, Esposito S, et al. Immune responses to a 2-dose schedule of the HPV-16/18 AS04-adjuvanted vaccine in girls (9-14) versus 3 doses in women (15-25): a randomised trial. Abstracts of the EUROGIN 2013 Congress, Florence, Italy, 3-6 November 2013; iW 1-5.

20. Goldie SJ, Grima D, Kohli M, et al. A comprehensive natural history model of HPV infection and cervical cancer to estimate the clinical impact of a prophylactic HPV-16/18 vaccine. Int J Cancer 2011; 106: 896-904.

21. Romanowski B, Schwarz T, Ferguson L, et al. Immune response to the HPV-16/18 AS04-adjuvanted vaccine administered as a 2-dose or 3-dose schedule up to 4 years after vaccination. Abstracts of the EUROGIN 2013 Congress, Florence, Italy, 3-6 November 2013; OC 6-11.

22. Dobson SR, McNeil S, Dionne M, et al. Immunogenicity of 2 doses of $\mathrm{HPV}$ vaccine in younger adolescents vs. 3 doses in young women: a randomized clinical trial. JAMA 2013; 309: 1793-1802.

23. Brotherton JM. Could one dose of bivalent HPV vaccine prevent cervical cancer? Lancet Oncol 2015; 16: 739-740.

24. Bednarczyk RA, Davis R, Ault K, et al. Sexual activity-related outcomes after human papillomavirus vaccination of 11- to 12-year-olds. Pediatrics 2012; 130: 798-805.

25. Muñoz N, Manalastas R Jr, Pitisuttithum P, et al. Safety, immunogenicity, and efficacy of quadrivalent human papillomavirus (types 6, 11, 16, 18) recombinant vaccine in women aged $24-45$ years: a randomised, double-blind trial. Lancet 2009; 373: 1949-1957.

26. Giuliano AR, Palefsky JM, Goldstone S, et al. Efficacy of quadrivalent $\mathrm{HPV}$ vaccine against HPV infection and disease in males. N Engl J Med 2011; 364: 401-411. 
27. Palefsky JM, Giuliano AR, Goldstone S, et al. HPV vaccine against anal HPV infection and anal intraepithelial neoplasia. N Engl J Med 2011; 365: 1576-1585.

28. Food and Drug Administration. Approval letter - GARDASIL 9. Silver Spring, MD: US Department of Health and Human Services, Food and Drug Administration; http://www.fda.gov/BiologicsBloodVaccines/Vaccines/ApprovedProducts/ucm426520.htm (access 2014.12.10).

29. Food and Drug Administration. Highlights of prescribing information. Gardasil 9 (human papillomavirus 9-valent vaccine, recombinant). Silver Spring, MD: US Department of Health and Human Services, Food and Drug Administration, 2014; http://www.fda.gov/downloads/Biologics-BloodVaccines/Vaccines/ApprovedProducts/UCM426457.pdf (access 2015.08.01).

30. Centers for Disease Control and Prevention (CDC). GRADE evidence tables - recommendations in MMWR. Atlanta, GA: US Department of Health and Human Services; http://www.cdc.gov/vaccines/acip/recs/ grade/table-refs.html (access 2015.03.27).

31. Joura EA, Giuliano AR, Iversen OE, et al. A 9-valent HPV vaccine against infection and intraepithelial neoplasia in women. N Engl J Med 2015; 372: 711-723.

32. Luxembourg A. Program summary and new 9-valent HPV vaccine trial data. Presentation before the Advisory Committee on Immunization Practices (ACIP), Atlanta, GA: US Department of Health and Human Services, CDC; http://www.cdc.gov/vaccines/acip/meetings/downloads/ min-archive/min-2014-10.pdf (access 2014.10.30).

33. Markowitz LE, Dunne EF, Saraiya M, et al. Centers for Disease Control and Prevention (CDC). Human papillomavirus vaccination: recommendations of the Advisory Committee on Immunization Practices (ACIP). MMWR Recomm Rep 2014; 63 (No. RR-05): 1-30.

34. Food and Drug Administration. Highlights of prescribing information. Cervarix [human papillomavirus bivalent (types 16,18 ) vaccine, recombinant]. Silver Spring, MD: US Department of Health and Human Services; Food and Drug Administration; http://www.fda.gov/downloads/ BiologicsBloodVaccines/Vaccines/ApprovedProducts/UCM186981.pdf. (access 2009).

35. Saslow D, Solomon D, Lawson HW, et al. American Cancer Society, American Society for Colposcopy and Cervical Pathology, and American Society for Clinical Pathology screening guidelines for the prevention and early detection of cervical cancer. J Low Genit Tract Dis 2012; 16: 175-204. 